\title{
LEVINAS ET LE SOCIALISME LIBERTAIRE
}

\author{
Cristobal Balbontin-Gallo ${ }^{\mathrm{I}}$
}

Résumé: Dans Autrement qu'être Levinas introduit la notion d'“an-archie”, pour désigner un ordre de sens immémorial aussi bien que dans un sens politique, qui fait de la place à une puissance de révolte issue de l'éthique. Désormais, la mise en place de l'ordre de sens politique ne permet plus d'englober ni d'épuiser la socialité issue de cette expérience intersubjective. Dans cette revendication anarchique de la socialité de l'humain Levinas rejoint donc quelques présuposés du socialisme et le judaïsme libertaire en Europe. Il s'agit donc de déceler les possibles points de raprochement et de croisement, qui aparaîtront d'une façon souvent inattendue pour une certaine orthodoxie levinassienne.

Mots clés: Socialisme. An-archie. Judaïsme. Libertaire. Levinas.

\section{INTRODUCTION}

Dans le texte La trace de l'autre paru dans la revue Tijdschrift voor Filosofie, de 1963, Emmanuel Levinas écrit:

La philosophie est atteinte, depuis son enfance, d'une horreur de l'Autre qui demeure Autre, d'une insurmontable allergie. C'est pour cela qu'elle est essentiellement une philosophie de l'être, que la compréhension de l'être est son dernier mot et la structure fondamentale de l'homme. C'est pour cela aussi qu'elle devient la philosophie de l'immanence et de l'autonomie ou, athéisme. Le Dieu des philosophes, d'Aristote à Leibniz, à travers le Dieu des scolastiques, est un dieu adéquat à la raison, un dieu compris qui ne saurait troubler l'autonomie de la conscience, se retrouvant elle-même à travers toutes ses aventures, retournant chez soi comme Ulysse

\footnotetext{
${ }^{1}$ Professeur auxiliaire de la Universidad Austral de Chile, Valdivia - Chile. Chercheur associé à l'Institut des Recherches Philosophiques (IRePh) de l'Université Paris-Nanterre. Docteur en Philosophie de l'Universität J.W. Goethe Frankfurt am Main et la Université Paris-Nanterre. (D) https://orcid. org/0000-0001-6604-2957. E-mail: cbalbonting@gmail.com.
}

https://doi.org/10.1590/0101-3173.2021.v44n4.07.p79 
qui, à travers toutes ses pérégrinations, ne va que vers son île natale. La philosophie qui nous est transmise attribue à ce retour non seulement la pensée théorétique, mais tout mouvement spontané de la conscience. Non seulement le monde compris par la raison cesse d'être autre car la conscience s'y retrouve, mais tout ce qui est attitude de la conscience, c'està-dire valorisation, sentiment, action, travail et, d'une façon plus générale engagement, est en derrière analyse conscience de soi, c'est-à-dire identité et autonomie. La philosophie de Hegel représente l'aboutissement logique de cette allergie foncière de la philosophie. (LEVINAS, 2001, p. 263).

Pourtant c'est bien aussi l'Etat hégélien qui est visé dans cette critique de l'économie totalisante qui mobilise la pensée métaphysique. En effet, chez Hegel, la médiation de l'État ne consiste qu'à s'extraire de la violence des points de vue particuliers pour laisser place au point de vue objectif et universel. Or pour Levinas il s'agit, au contraire, d'une violence faite à l'autre au nom d'un système de la totalité qui cherche à englober la singularité concrète de l'homme dans l'universel. D'où le fait que la marche de l'Etat ne puisse être séparée de la marche de la Raison dans l'histoire, qui cherche sur son parcours à surmonter toute différence particulière dans le logos. Dès lors, la réalité concrète des individus ne devient que le terrain de mise à l'épreuve du rationnel jusqu'à sa réalisation ultime où tous les points de vue sont synthétisés dans l'État et où l'universel fait du particulier une ruse pour revenir à lui-même. Il s'agit en arrière-plan de la réalisation libre de l'esprit comme autosuffisant à lui-même, où l'accomplissement métaphysique de l'ensemble du réel dans le rationnel n'est autre chose que le mouvement de la vérité, de la mise en adéquation de l'être et la pensée.

Or, malgré cette critique de l'Etat, dans Autrement qu'être ou au-delà de l'essence Levinas fait de la place au politique par l'entremise de la figure du tiers. Cependant cette mise en place du politique chez Levinas ne retranche en rien les mesures de prévention et les avertissements faits par le philosophe dans Totalité et infini à l'égard du lexique totalisant qui caractérise le politique. Ainsi, dans Autrement qu'être Levinas introduit aussi la notion d'an-archie, non seulement pour faire référence à un ordre de sens immémorial dans la trace de l'autre en soi, mais aussi pour faire référence à l'anarchie dans un sens politique qui revendique un désordre, voire une puissance de révolte issue de la socialité éthique que l'expérience du visage réveille à l'égard de la politique. Désormais, la mise en place de l'ordre de sens politique ne permet plus d'englober ni d'épuiser la socialité issue de cette expérience intersubjective. Dans cette 
revendication an-archique de la socialité de l'humain face à l'abstraction de l'homme dans des institutions objectives du social, Levinas rejoint donc quelques présuposés du socialisme libertaire, dont il s'agit de déceler les points de raprochement et de croisement, qui aparaîtront d'une façon inattendue pour une certaine orthodoxie levinassienne.

\section{LeVINAS CRITIQue de L'ETAT}

Un des sujets centraux dans l'Etoile de la rédemption de Franz Rosenzweig est la condamnation radicale de la dimension politique de l'histoire chez Hegel. Ce sujet central a-sans doute- son origine dans la thèse de doctorat de Rosenzweig Hegel et l'État. C'est aussi bien dans l'analyse de la Phénoménologie de l'esprit que des Principes de la philosophie du droit de Hegel, que Rosenzweig trouve sa source d'inspiration. La consolidation de la Realpolitik dans l'Allemagne de la fin du $19^{\text {ème }}$ siècle, mettant en scène le triomphe du machiavélisme politique, coïncide avec le génie fondateur de Bismarck, et trouve sa réminiscence dans le mouvement hégélien qui veut réconcilier les particularités de chaque Land dans un universalisme eidétique qui mobilise l'inspiration nationale allemande. C'est justement Meinecke, maitre de Rosenzweig, qui estime que le trait dominant de l'État est le nationalisme hérité de la révolution française au-delà des particularités locales. Dès lors, la dimension absolue de l'État constitue une fin en soi où les particularités se veulent absorbées dans le corps étatique afin d'être réalisées. Jusqu'à ce point, aucune de ces circonstances prises seules ne paraissent justifier la critique de l'État hégélien par Rosenzweig. Mais toutes ces circonstances réunies simultanément avec l'événement tragique de la guerre de 1914-1918 expliquent le ressort matériel de la critique. Concrètement, ce sera l'épreuve angoissante de la guerre vécue par Rosenzweig dans les tranchées des Balkans, un événement crucial, qui guidera l'horizon de ses réflexions. En effet, il s'agit de dénoncer la violence impersonnelle de l'État et le sacrifice de l'individu dans le système de la totalité. Désormais, l'effort de Rosenzweig sera de démontrer la continuité entre la réalisation de cette totalité absolue et la violence contre les individus. Il s'agit d'une violence faite à l'homme au nom de tout le social dans l'État. Cette violence intervient à l'intérieur même de la vie politique des États qui avancent dans l'histoire à travers des guerres et des révolutions. Dès lors, l'État ne peut être séparé de la marche de la Raison hégélienne dans lhistoire, où cette Raison déploie son processus. Ce faisant, Rosenzweig établit une connexion directe entre la dernière section de la Phénoménologie de l'esprit et les Principes de la philosophie du droit. Dès lors, dans 
l'interprétation de Rosenzweig de l'architecture hégélienne, si l'État fait partie intégrante du système de l'esprit absolu, on comprend bien que l'État lui-même est pris dans un système de médiation d'ordre ontologique présuposé à l'arrière plan. Ainsi, bien avant Heidegger, Rosenzweig voit dans la philosophie de Hegel l'accomplissement de la métaphysique qui cherche à englober l'ensemble du réel dans le rationnel, dans une conscience de soi qui s'élève au rang d'universel dans l'État. par exemple:

Hegel paraît confirmer cette perspective dans différents passages. Ainsi,

L'idée de l'Etat: a) a une effectivité immédiate et est l'Etat individuel en tant qu'organisme qui est en relation avec soi - constitution ou droit étatique interne; b) elle passe au raport de l'Etat singulier avec des autres Etats - droit étatique externe; c) elle est l'idée universelle en tant que genre et puissance absolue à l'encontre des Etats individuels, [elle est] l'esprit qui se donne son effectivité dans le procès de l'histoire du monde. (HEGEL, $1998, \$ 259)$.

En effet, l'agencement rationnel du tout et des parties permet de mesurer la séparation qui existe entre la totalité comme catégorie logique et la réalité concrète qu'elle englobe et qui soutient cette totalité. C'est la correspondance des parties avec le tout que contient aussi la téléologie de l'histoire comme unité des événements, du monde et de la pensée. L'histoire englobe la pensée et la pensée finit par englober l'histoire. L'unité avec l'essence relève l'histoire au niveau de destin. Cette réalité concrète est l'histoire universelle: le terrain de mise à l'épreuve du rationnel. Alors l'histoire universelle doit être comprise dans sa forme ultime: l'État. Ainsi, Hegel (1988, semble confirmer cette perspective dans le paragraphe 549 de la troisième partie de l'Encyclopédie des sciences philosophiques (intitulée Philosophie de l'esprit) en signalant:

Ce mouvement est le chemin de la libération de la substance spirituelle, l'acte par lequel le but final absolu du monde s'accomplit en celui-ci, par lequel l'esprit qui n'est d'abord qu'en soi s'élève à la conscience et à la conscience de soi, et, par là, à la révélation et effectivité de son essence étant en et pour soi, et, par lequel il devient à lui-même aussi esprit extérieurment universel, esprit du monde. En tant que ce dévelopement est dans le temps et dans l'être-là, et par là, en tant qu'histoire, ses moments et degrés singuliers sont les esprits-des-peuples ; chacun [de ceux-ci], en tant qu'esprit singulier et naturel dans une déterminité qualitative, est déterminé à n'occuper qu'un seul degré et à n'accomplir qu'une seule tâche de l'acte total. 
Or, dans Totalité et infini Levinas est à peu près d'accord avec la critique que Rosenzweig porte contre l'État chez Hegel. La préface de Totalité et infini rapelle combien l'ouvrage de Rosenzweig était important pour Levinas: "L’oposition à l'idée de totalité, nous a frapé dans le Stern der Erlösung de Franz Rosenzweig, trop souvent présent dans ce livre pour être cité." (LEVINAS, 1961, p. 14). Ainsi, l'État hégélien qui veut réconcilier les hommes, finit par les neutraliser dans l'universel et implique leur résignation à l'anonymat. "La tendance naturelle de l'instance étatique" nous dit Levinas "est portée à s'autonomiser par raport aux moments ou aux éléments desquels elle procède." (LEVINAS, 1991a, p. 105). C'est une forme d'inhumanité où la voix des personnes singulières se heurte à une surdité, où elle disparaît au profit d'une généralité. C'est une perspective de la paix qui depuis Platon veut entendre la paix comme une réconciliation des perspectives dans une vérité commune qui s'autonomise pour surmonter tout conflit venu et à venir. Il faut donc - d'après Levinas - relativiser l'ordre de la rationalité objective, où les cris des victimes qui en apellent avec insistance aux jugements des juges et des hommes d'État sont dissimulés sous les identités de citoyens (LEVINAS, 1991b, p. 126 ss). Levinas (2006, p. 125) signale encore:

C'est précisément le destin de la philosophie occidentale et sa logique de se reconnaître une condition politique, au point que la pleine expression de la vérité et la constitution de l'État universel, à travers les guerres et les révolutions coïncident. Les heurts entre hommes [dont la lutte pour la reconnaissance], l'oposition des uns aux autres, l'oposition de chacun à soi, font jaillir des étincelles d'une lumière ou d'une raison qui domine et pénètre les antagonismes. La vérité ultime qui embrasse toutes ces étincelles comme la fin de l'histoire embrasse toutes les histoires. Les deux événements ne font qu’un.

Ainsi, cette coïncidence révélée dans l'État comme institution de la Sittlichkeit porte en plus une référence critique à cette liberté en vue de la liberté, ou tautologie de la liberté, qui risque d'aboutir à une tyrannie. "L'État qui réalise son essence à travers les œuvres, glisse vers la tyrannie et atteste ainsi mon absence de ces œuvres qui me reviennent étrangères à travers les nécessités économiques." (LEVINAS, 1961, p. 151). D'où le fait “[...] qu'il y a des avatars qui sont terribles, parce qu’ils proviennent précisément de la nécessité de l'ordre raisonnable. Il y a, si vous voulez, des larmes qu'un fonctionnaire ne peut pas voir: les larmes d'autrui." (LEVINAS, 1991a, p. 105). Levinas signale de même: 
Mais apercevoir dans ce discours, dans cette possibilité de parler, conquise à partir de la pensée totalisante, une lointaine impossibilité du discours -l'ombre du soir dans le soleil du plein midi- ; pressentir, à travers cette philosophie de la totalité qui détend l'égoïsme subjectif (fût-il sublime comme la soif du salut), la fin de la philosophie aboutissant au totalitarisme politique où les hommes ne sont plus la source de leur langage, mais le reflet du logos impersonnel ou rôles joués par des figures, tout cela constitue la valeur de la notion kierkegaardienne de l'existence et sa protestation foncièrement protestante contre les systèmes. (LEVINAS, 1976, p. 80).

C’est ainsi que Levinas (1961, p. 276) conclut: "La politique laissée à elle-même porte à une tyrannie."

Or cette critique peut être double avec l'exégèse que Levinas fait du Talmud juif. En effet, dans l'une de ses lectures talmudiques qui se trouve dans le recueil L'Au-delà du verset intitulée "L'État de César et l'État de David" de 1971, Levinas laisse place au politique sous la figure romaine de "l'État de César". Mais ceci ne saurait être sans référence à "l’État de David". En effet, et à propos de l'État de César, Levinas reprend des critiques que l'on a par ailleurs examinées à propos de l'État comme totalité. Certes, l'État de César se caractérise par l'imposition d'un ordre public, mais qui peut toujours dégénérer dans la violence de la totalité. Succinctement, il s'agit d'un État unidimensionnel centré sur lui-même qui trouve son centre de gravité en lui-même et qui peut, dès lors, virer en totalitaire. C'est bien encore la dénonciation d'une logique centripète où l'État a "raison d'État" qui l'amène à sombrer dans la persévérance de son propre être. Comme le dit Levinas: "Il sépare l'humanité de sa délivrance. Incapable d'être sans s'adorer, il est l'idolâtrie elle-même."' (LEVINAS, 1982, p. 216). Comme l'exprime à son tour Catherine Chalier, "État romain, modèle de rationalité de pouvoir, d'une politique nécessaire mais aspirée vers la tyrannie."3 (CHALIER, 1998, p. 119). Désormais, si Levinas reconnaît ici une certaine valeur à l'État, chez lui cette reconnaissance n'est en aucun cas "inconditionnelle". Autrement dit, si Levinas laisse de la place dans sa réflexion talmudique à l'État, c'est toujours en vue d'un dépassement dans un "au-delà de l'État".

${ }^{2}$ LEVINAS, E. Au-delà du verset, op. cit., p. 216.

${ }^{3}$ CHALIER, C. Exposé dans le colloque Difficile justice. Dans la trace d'Emmanuel Levinas, op. cit., p. 119. 


\section{L'ANARCHISME NECESSAIRE}

Pourtant dans Autrement qu'être ou au-delà de l'essence Levinas fait de la place dans sa philosophie au politique. En effet, dans le schéma de Totalité et infini, le visage est compris comme visage des visages -c'est-à-dire- comme l'humanité toute entière. Ce faisant il n'y a pas de place pour l'intervention des institutions au bénéfice de l'égalité de tous, du fait que la justice est réinterprétée à partir de l'inégalité de l'un-pour-l'autre. Or dans Autrement qu'être, le tiers n'est pas seulement l'autre de l'autre mais aussi l'autre que l'autre. Alors la responsabilité à l'égard d'autrui doit aussi prendre en charge la responsabilité du tiers. Désormais il faut la mise en place d'un deuxième ordre de sens, où la responsabilité extrême doit faire place aussi à la justice. Ce faisant, comme Levinas (1974, p. 205) lui même l'exprime: "L'extraordinaire engagement d'autrui à l'égard du tiers en apelle au contrôle, à la recherche de la justice, à la société et à l'État [...] et hors de l'anarchie, à la recherche d'un principe." Ou encore:

A ma relation avec l'unique et l'incomparable se superpose la comparaison et, en vue d'équité ou d'égalité, une pesée, une pensée, un calcul, la comparaison des incomparables, et, dès lors, la neutralité -présence ou représentation de l'être, [...] et par là l'importance extrême dans la multiplicité humaine de la structure politique de la société soumise aux lois et dès lors aux institutions où le pour-l'autre-de-la-subjectivité -le moi- entre avec la dignité du citoyen dans la réciprocité parfaite des lois politiques essentiellement égalitaires ou tenues à le devenir. (LEVINAS, 1984, p. 345).

"Les institutions et l'État lui-même peuvent être retrouvés à partir du tiers intervenant dans la relation de proximité." (LEVINAS, 1993, p. 211). C'est donc un recours à la société aussi bien qu'à un ordre politicoinstitutionnel que la justice à l'égard du tiers permet.

Cependant cette mise en place de la société et du politique chez Levinas ne retranche pas les mesures de préventions et les avertissements faits par le philosophe déjà dans Totalité et infini à l'égard du lexique totalisant qui caractérise le politique ainsi que la dimension objective du social. Avec ce propos Levinas parle d`une an-archie pas seulement pour relever la trace de l'autre en soi, qui fait signe dans l'un pour l'autre de la responsabilité, mais aussi dans un sens politique qui -comme on l'avait déjà indiqué-revendique 
un désordre nécessaire. Ainsi tout l'enjeu chez Levinas consiste à la fois à laisser place au politique, mais à se prémunir de son économie totalisante.

En effet, Levinas utilise continûment, depuis Humanisme de l'autre homme et surtout dans Autrement qu'être l'expression an-archie. D'abord, dans un sens immémorial lié à la trace de l'Autre en nous: "La première pourrait être apelée le 'recul à l'en-deçà': en deçà du commencement, de l'arché [...] arché d'une requête qui fait trace de je ne sais où.” (LEVINAS, 1974, p. 127). Pourtant, par la suite, Levinas, lui, désigne expressément un usage politique. Ainsi écrit-t-il: "[...] elle ne peut que troubler -mais d'une façon radicale- et qui rend possible des instants de négation sans aucune affirmation -l'État. L'État ne peut s'ériger en tout [...]. Le désordre a un sens irréductible en tant que refus de synthèse." (LEVINAS, 1974, p. 128 n.3). Passage à la suite duquel Jacques Rolland (2000, p. 207) remarque:

Trouble que l'on sait par ailleurs synonyme de tumulte qui 'sème le trouble' dans l'ordre institué [...]. Trouble qui réhabilite ainsi l'emploi le plus populaire et le plus immédiat du mot anarchie, laissant aparâtre qu’il n'y aurait pas d'an-archie sans [...] une certaine anarchie.

Cette référence à l'anarchie politique sans laquelle il n'y aurait pas d'an-archie métaphysique semble être confirmée par Levinas lui-même. Concrètement, dans Totalité et infini, avant les dévelopements du concept d'an-archie dans Humanisme de l'autre homme, dans le chapitre IV d'Autrement qu'être et dans le cours "La subjectivité comme anarchie" contenu dans le recueil Dieu, la mort et le temps, publié sous les soins de Jacques Rolland en 1993, on constate déjà les premières aparitions du mot pour décrire la résistance de la multiplicité humaine à l'ordre de la totalité. Levinas écrit (1961, p. 270): “Les moi's ne forment pas de totalité. Il n'existe pas de plan privilégié où ces moi's pourraient se saisir de leur principe. Anarchie essentielle à la multiplicité". Passage qui rejoint l'esprit du texte Le temps et l'autre en ceci: "C'est, au contraire, vers un pluralisme qui ne se fusionne pas en unité que nous voudrions nous acheminer." (LEVINAS, 1983, p. 20).

Pourtant il ne s'agit pas d'abandonner l'État pour faire place à l'anarchisme. De même que dans Autrement qu'être l'éthique ne veut pas être comprise comme ontologie -fût-elle ontologie première-, donc posée comme premier principe. Il ne s'agit pas non plus de poser l'anarchie comme système politique car cela viendrait contredire le sens même de l'an-archie. Sans se placer en continuité ni commencement mais plutôt comme une irruption, 
voire une disruption, il s'agit plutôt de maintenir la place de l'anarchie à côté du politique et non de substituer l'un à l'autre. Du coup, l'anarchie pour Levinas est le plus grand désordre des désordres parce que non oposée ni oposable à l'ordre comme alternative ou comme jeu de possibilités. Levinas parlera d'un "[...] désordre ou révolution permanente, rupture des cadres, effacement des qualités, et, telle la mort, le libérant de tout et du tout; comme si l'autre homme était recherché -ou aproché- dans une altérité où aucune administration ne pourrait jamais l'atteindre." (LEVINAS, 1992, p. 27). Dès lors, bien que résistance et révolte, l'anarchie signifie aussi l'inspiration de la conscience, l'ouverture. Une ouverture qui est aussi ouverture du politique à un au-delà du politique, c'est-à-dire, une ouverture à travers l'éthique issue de la socialité, donc de l'expérience concrète du visage face-à-face. Alors il revient à l'anarchie (éthique) d'être aussi une source drinspiration du politique quelle a mis en question. Cette source vive c'est la fraternité, l'apartenance de l'humain à l'humain. Ce que Levinas apelle la "proximité".

\section{LEVINAS ET LE SOCIALISME LIBERTAIRE}

Pour Levinas, il s'agit donc de reconduire la politique non vers la liberté mais vers la fraternité. La liberté individuelle est considérée comme solidaire de l'ontologie dont il s'agit de sortir au bénéfice de la relation exceptionnelle d'un homme avec l'autre homme qui le précède. Avec ce propos, Levinas emprunte de façon successive la phrase suivante de Pascal pour caractériser cette perspective critique de l'individualisme:

Mon "au-monde" ou ma "place au soleil", mon chez-moi, n'ont-ils pas été usurpation des lieux qui sont à l'autre homme déjà par moi oprimé ou affamé ? Citons encore Pascal: "C'est ma place au soleil, voilà le commencement et l'image de l'usurpation de toute la terre." Crainte pour tout ce que mon exister, malgré son innocence intentionnelle et consciente, peut accomplir de violence et de meurtre. Crainte qui remonte de derrière ma "conscience de soi" et quels que soient vers la bonne conscience les retours de la pure persévérance dans l'être. (LEVINAS, 1991b, p. 139).

Or, Levinas n'est pas le premier penseur à s'inspirer de Pascal. Avant Levinas, Rousseau s'est nourri de cette perspective.

En effet, Rousseau désigne, dans un passage qui a une véritable force prophétique, l'origine du mal: 
Le premier qui, ayant enclos un terrain, s'avisa de dire: Ceci est à moi, et trouva des gens assez simples pour le croire, fut le vrai fondateur de la société civile. Que de crimes, de guerres, de meurtres, que de misères et d'horreurs n'ê̂t point épargnés au genre humain celui qui, arrachant les pieux ou comblant le fossé, ê̂t crié à ses semblables: Gardez-vous d'écouter cet imposteur ; vous êtes perdus, si vous oubliez que les fruits sont à tous, et que la terre n'est à personne. (ROUSSEAU, 1987, p. 59).

Or, cette phrase n'est pas n'importe quelle phrase. Elle ouvre en 1755 - comme le rapelle Michael Lowy - un nouveau chapitre dans l'histoire de la pensée: le chapitre du romantisme. C'est dans la Nouvelle Héloise qu'il confirmera cette perception avec le tableau d'une société composée d'êtres seuls, incompris, abandonnés et incapables de communiquer d'une manière significative avec leurs semblables. Peu importe donc que l'auteur du Discours ait changé d'avis, qu'il y ait d'autres écrits où il défend la propriété privée, et avance des propositions prudentes de réforme institutionnelle. Sur ce point, on rejoint plutôt Bousquet pour qui “[...] tout Rousseau n'est pas romantique mais à peu près tout le romantisme est déjà chez Rousseau.” (BOUSQUET, 1981, p. 194). Ce faisant, Rousseau aurait inauguré une longue tradition de pensée pourfendeuse d'un socialisme à contre courant de la modernité.

En effet, depuis Rousseau une longue ligne de penseurs a nourri ce foyer de pensée constituant une critique de la réalité sociale existante au nom d'un autre monde possible. C'est en effet aussi le cas de Lukacs qui, dans sa pensée anticapitaliste, vise à oposer ce nouveau courant de pensée à certains thèmes centraux de la modernité comme l'individualisme économique et la rationalité technique. Ce faisant, il rejoint la constatation de Max Weber, pour qui l'une des principales caractéristiques de la modernité sont l'esprit de calcul, la rationalité bureaucratique, voire la domination bureaucratique. Toutes ces caractéristiques sont à leur tour, non séparables du capitalisme. Pour Lukacs, l'industrialisation, le dévelopement rapide des sciences et de la technologie, l'hégémonie du marché, la division du travail et la conséquente abstraction des raports humains sont le signe d'un nouveau système économique. Autrement dit, il s'agit de ce que la théorie critique apelle, d'après Lukacs, la réification. Cette réification aparaît comme une force d'un côté à travers les nouveaux moyens de production mis en place et, de l'autre à travers l'État et l'apareil politique moderne qui gère ce nouveau système social (LUKACS, 1960). Il s'agit d'un nouveau système qui trouve -dans le projet de dévelopement du sujet individuel-, son accomplissement dans un individu isolé. De cette manière, 
la revendication d'une conception relationnelle de l'individu est essentielle au socialisme. Ainsi pour cette pensée critique, l'individu est la conscience malheureuse d'une coupure des liens humains cherchant à les restaurer. Il en va de même pour Marx dans le Manifeste du Parti communiste lorsqu'il critique “[...] l'eau glaciale du calcul égoïste qui caractérise la bourgeoisie." (1895, p. 7). Portée impersonnelle et mécanique présente pour Marx dans le système capitaliste qui est fondé sur un système de catégories abstraites: le travail abstrait, la valeur abstraite des échanges, les moyens et raports impersonnels de production. C'est ce qui implique de subordonner aux lois économiques la substance de la société elle-même. Pour sa part, Engels, dans un passage saisissant de la Condition de la classe ouvrière en Angleterre remarque:

Les centaines de milliers de personnes de tous rangs et classes qui s'[y] basculent, ne sont-elles pas toutes des êtres humains avec les mêmes qualités et potentialités, et avec le même intérêt d'être heureux ?.. Et cependant elles se côtoient comme si elles n'avaient rien en commun, rien à faire les uns avec les autres... Cette indifférence brutale, cet isolement insensible de chaque personne dans son intérêt privé devient d'autant plus répugnant et offensif quand ces individus s'entassent dans un espace limité. (ENGELS, 1987, p. 17).

La rationalité instrumentale et la rationalité bureaucratique sont pour Max Weber au cour de la civilisation bourgeoise moderne. Pour Adorno et Horkheimer dans la Dialectique des Lumières, il s'agit d'oposer à ce rationalisme abstrait une autre forme de rationalité humaine substantielle. En oposition à l'abstraction rationnelle, il s'agit donc d'après Marx d'un retour au concret. Ceci implique donc une revalorisation de l'intuition au-dessus de l'activité judicative de l'esprit. En outre, il faudra considérer le mouvement de Mai 68 qui remet en question la modernisation capitaliste, la société de consommation et la technocratie, et dont fait partie le socialisme libertaire contemporain dans sa révolte contre l'unidimensionnalité de l'homme issu de la société industrielle. Il en va de même pour la Théologie de la libération en Amérique latine qui, dans un autre registre et avec une intention autre, se veut aussi une critique de la modernité capitaliste et dénote la nostalgie d'une forme de communauté organique à travers son aspiration à une société égalitaire et sans classes.

Or, face à la rationalisation instrumentale issue de la modernité qui conduit à bannir l'humain de la sphère publique et à établir des raports purement utilitaires, Max Weber parle de la recherche d'un refuge dans “[...] la 
fraternité des relations directes et réciproques entre individus isolés." (WEBER, 1963, p. 96). C'est la constatation sombre de la réalité d'une époque où l'usine capitaliste aparaît comme un lieu infernal pour les ouvriers. Rythme uniforme et mécanique qui condamne les travailleurs à être une masse anonyme d'êtres semblables, sans différences. Dans un espace-temps qui a perdu toute diversité qualitative comme toute variété pour devenir régulier, uniforme et prévisible. Comme le signale Thomas Carlyle dans son texte Signe des temps: "[...] les êtres humains eux-mêmes sont devenus mécaniques dans leur tête et leur cœur, en même temps que dans leurs mains." (1829, p. 441). Vie sociale, religion, la politique devient scandée par cette logique mécanisante. Sans aller aussi loin, quelques penseurs considèrent l'État moderne et son administration bureaucratique comme précisément fondés sur cet individualisme abstrait ; assimilables à une institution aussi mécanique et impersonnelle qu'une usine. Déjà, Novalis signalait que “[...] dans aucun État l'administration n’a été aussi parfaitement semblable à une fabrique qu'en Prusse depuis la mort de FrédéricGuillaume II.” (DROZ, 1963, p. 61, 86, 169). Pour sa part, Friedrich Schlegel se plaint à son tour des risques qu'implique une "[...] certaine conception mathématique de l'État.” (DROZ, 1963, p. 169).

Or, parmi ces références à la pensée libertaire, on doit particulièrement prendre en considération le romantisme utopique de Buber et Rosenzweig dont on sait combien leurs pensées étaient fort importantes pour Levinas. ${ }^{4}$ C'est à travers Gustav Landauer, l'un des représentants les plus typiques du socialisme libertaire, que Buber trouve une inspiration décisive. Mais, dans une plus large mesure, c'est dans la constellation du judaïsme libertaire d'Europe centrale que sa pensée politique doit être située. ${ }^{5}$ Déjà dans l'écrit Gemeinschaft, de 1919,

\footnotetext{
${ }^{4}$ L'influence de Buber sur Levinas est remarquable. Levinas dédie plusieurs articles et fait des références explicites à Buber dans différents ouvrages parmi lesquels: Noms Propres, les préfaces aux éditions allemandes de Totalité et infini aussi bien que dans Hors sujet, sans considérer l'importante correspondance entre les deux penseurs dont une partie reste inédite. Par raport à Rosenzweig, la préface de Totalité et infini rapelle combien il était fort important pour Levinas. "L'oposition à l'idée de totalité nous a frapé dans le Stern der Erlösung de Franz Rosenzweig, trop souvent présent dans ce livre pour être cité." (1961).

${ }^{5}$ Le terme Europe centrale - comme le décrit Michael Löwy dans son livre Le judaïsme libertaire de l'Europe centrale - "[...] désigne dans sa plus large mesure une aire géographique, culturelle, historique unifiée par la culture germanique, c'est-à-dire l'Allemagne et l'empire austro-hongrois. Pendant la période qui s'étend du milieu du XIX ${ }^{\text {ème }}$ siècle jusqu’à 1933, la communauté juive d'Europe Centrale a connu une floraison culturelle extraordinaire, un siècle d'or comparable au XII ${ }^{\text {ème }}$ siècle judéo-arabe en Espagne. Cette culture judéo-allemande, produit une synthèse spirituelle unique en son genre qui a donné au monde Heine et Marx, Freud et Kafka, Ernst Bloch et Walter Benjamin parmi d'autres, nous aparaît aujourd'hui, comme un monde disparu, un continent effacé de l'histoire. Détruite par le nazisme, elle n'a survécu qu'en exil, dispersée, et ses derniers représentants Marcuse, Fromm, Bloch
} 
Buber critique l'État mécaniciste qui veut se substituer à la vie organique du social. Puis, c'est surtout dans Socialisme et utopie qu'il dévelopera les traits principaux de son socialisme libertaire à travers une relecture de la tradition socialiste utopique (Fourier, Saint-Simon, Owen), anarchiste (Proudhon, Kroptotkine, Landauer) ainsi que marxiste (Marx, Engels, Lenine). Le cour de son argument est une critique radicale de l'État capitaliste moderne qui aurait brisé la structure intersubjective de la société. Le nouveau capitalisme centralisé aurait ainsi réussi à faire ce que l'ancien État despotique n’aurait pu accomplir: l'atomisation de la société. Pour Buber, le capitalisme ne veut faire face qu'à des individus. A son tour, l'État moderne se met à son service afin de déposséder progressivement la vie des groupes de leur autonomie. Ainsi, l'essence communautaire de la vie sociale se trouve progressivement vidée par les contraintes économiques ainsi que par l'État capitaliste. Comme le signale Levinas à propos de Buber, "Il suit une idée: c'est dans l'oposition du politique et du social qu'il [Buber] situe les doctrines étudiées. Comme s'il s'agissait de mettre en question la subordination de la société civile à l'État où, pour Hegel, l'humanité atteindrait à l'universalité de la pensée et du vouloir csest-à-dire à la liberté." Il continue son cheminement réflexif en affirmant: "C'est à partir de l'idée de domination, de la coercition -ou comme on dirait aujourd'hui de la répression- que Buber pense le raport politique entre hommes." (LEVINAS, 1977 a, p. 8-9). Il s'agit pourtant pour Buber justement du contraire: de remplacer l'État par la société. La polémique avec Hermann Cohen témoigne de cette priorité: "L'humanité - et dire cela monsieur le professeur Cohen- est en ce moment plus grande que l'État. Cohen veut soumettre l'Esprit à l'État, moi l'État à l'Esprit." (BUBER, 1916, p. 427-428). Mais cela exige que la société ne soit plus un ensemble formé uniquement d'individus. La société authentique qui remplace l'État doit être une pluralité riche d'associations. Cela implique un changement qualitatif de relations humaines, aussi bien qu'un changement face au capitalisme et à l'État. La nouvelle forme sociale organique serait la renaissance du social sous la forme d'une fédération décentralisée de petites communautés. Il ne fait pas mystère que Buber a entrevu cette possibilité dans la révolution de la Commune de Paris et surtout dans les kibboutzim. Or, il s'agit surtout de concevoir une collectivité humaine hors des raports de domination et de la logique de pouvoir. Il n'est donc pas

sont déjà partis. Néanmoins, elle a laissé son empreinte sur la culture du vingtième siècle, dans ce qu'elle a produit de plus novateur et riche dans les sciences, la littérature ou la philosophie." Cf. LÖWY, Michael. Le judaïsme libertaire en Europe central. Paris: du Sandre, 2009, p. 7. 
étonnant que d'après le texte Gemeinschaft, Buber se réclame de Kropotkine, Tolstoi et Landauer pour condamner la tyrannie de l'État.

Quant à Rosenzweig, suivant les indications de Günther Henning, c'est celui qui "[...] plus que tout autre, a transposé les intentions du romantisme dans une philosophie systématique de la religion.” (HENNING, 1974, p. 45). Cela vaut d'abord pour sa critique de la conception moderne du temps comme progrès. En outre, par raport au capitalisme, Rosenzweig signale en 1919 qu’il est un système "dominé par l'esclavage" et que le chemin de l'émancipation est "[...] l'abandon du marché libre." (ROSENZWEIG, 1937, p. 470). Pour ce qui est dit à propos de l'État, dans L'étoile de la Rédemption, Rosenzweig signale que la violence, et non le droit, est le vrai visage de l'État (ROSENZWEIG, 1982, p. 92-95). Ce qui l'amène à défendre une anhistoricité et un caractère apolitique radical du peuple juif. Un peuple qui, désormais, ne saurait être impliqué dans la violence de l'histoire des États. Rester juif, c'est donc pour lui refuser l'État, c'est résister au vent de l'histoire qui emporte dans la violence.

Or, la pensée de Levinas n'est pas étrangère à l'inspiration du socialisme libertaire. On retrouve cette inspiration libertaire depuis ses premières lectures de l'écrivain romantique Tolstoï. De même, la pensée de Levinas n'est pas loin des penseurs ici évoqués, à l'exception de Rousseau dont Levinas n'était pas un lecteur assidu. D'abord, à plusieurs reprises il confirme son affinité avec le marxisme à propos duquel il écrira:

Non, dans le marxisme il n'y a pas que la conquête; il y a la reconnaissance de l'autre. Certes, il consiste à dire: l'autre, nous pouvons le sauver si l'autre réclame ce qui lui est dû pour lui-même. Le marxisme invite l'humanité à réclamer ce qu'il est de mon devoir de lui donner. C'est un peu différent de ma distinction radicale entre moi et les autres mais le marxisme ne peut pas être condamné pour cela. Non parce qu'il aurait tellement réussi, mais parce qu'il aurait pris l'Autre au sérieux. (LEVINAS, 1991b, p. 130).

De même lorsqu'il écrit: "Le marxisme, dont il serait injuste de méconnaître l'originelle amitié pour l'autre homme, méfiant à l'égard d'une philanthropie de pure inspiration - sans devoirs, sans contraintes, sans les droits de l'autre homme [...]." (LEVINAS, 1990, p. 114). Or, la familiarité du marxisme avec Levinas ne fait pas mystère, même s'il condamne le stalinisme. ${ }^{6}$ De son vivant Levinas a été le témoin direct de la révolution russe et il s'est montré solidaire de cette inspiration de jeunesse. Ainsi, Levinas déclare: "Je ne

${ }^{6}$ Entretien: Philosophie, Justice, amour. In: Entre nous. Essai sur penser à-l'autre. Paris: Grasset, 1991. 
suis pas resté indifférent aux tentations de la révolution léniniste, au monde nouveau qui allait venir. Mais sans engagement de militant." (POIRIER, 1996, p. 68). Jeune il se serait aussi familiarisé par l'entremise de Halbwachs, à l'Université de Strasbourg, avec les principaux théoriciens du socialisme: Saint-Simon, Fourier, Proudhon, Marx, Sorel (LESCOURRET, 1994, p. 55). Postérieurement dans son enseignement, il reviendra dans les années 1964-1965 sur la pensée socialiste à l'université, où il assure un cours sur les principaux théoriciens du socialisme (LESCOURRET, 1994, p. 229).

Levinas rejoint aussi la pensée de Marx sur l'idée d'un temps prometteur comme il le dit dans un entretien tout à fait intéressant publié lors de l'écroulement du mur de Berlin:

Depuis la Bible, nous sommes accoutumés à penser que le temps va quelque part, que l'histoire de l'humanité se dirige vers un horizon, même à travers des détours ou des vicissitudes. L'Europe a bâti sa vision du temps et de l'histoire sur cette conviction et cette attente: le temps promettait quelque chose. Malgré son refus de la transcendance et de la religion, le régime soviétique était l'héritier de cette conception. Depuis la révolution de 1917 on avait le sentiment que quelque chose continuait à s'annoncer, à se préparer en dépit des obstacles ou des erreurs. Avec l'effondrement du système soviétique [...] le trouble atteint des catégories très profondes de la conscience européenne. Notre raport au temps se trouve mis en crise. (LEVINAS, 1992b, [s.p]).

En outre, par raport à la critique de l'économie capitaliste, Levinas est aussi solidaire du marxisme et critique le risque qu'implique de laisser l'économie à son propre déterminisme. Levinas écrit:

Notre vieux texte affirme le droit de la personne, comme de nos jours l'affirme le marxisme, le marxisme humaniste [...] qui se demande: "Comment l'homme, ami de l'homme, a pu dans des conditions déterminées, se faire ennemi de l'homme ?", et pour qui cette anomalie que l'on apelle aliénation s'explique par la structure de l'économie, laissée à son propre déterminisme. Notre Michna entend aussi imposer une limite à l'arbitraire de l'économie et à cette aliénation. (LEVINAS, 1977b, p. 17).

D'ailleurs Levinas condamne la dimension impersonnelle et d'aliénation abstraite des travailleurs œuvrant dans la production collective: 
Le tiers saisissable à partir de son ouvre -à la fois présent et absent-, sa présence à la troisième personne marque exactement la simultanéité de cette présence et de cette absence. Il est livré à mon pouvoir en tant qu'extérieur à ma prise. Il est accessible dans l'injustice. Et c'est pourquoi l'injustice -à la fois reconnaissance et méconnaissance- est possible par l'or qui force et tente, instrument de la ruse. L'injustice par laquelle le moi vit dans une totalité est toujours économique. (LEVINAS, 1991b, p. 40).

Le visage devient anonyme dans une totalité d'ordre économique. D’où le fait que Levinas affirme que l'on se soumet à l'exigence "de transcender, en quelque façon, ce que la nature peut comporter d'inhumain et le corps social de nécessités aveugles" (LEVINAS, 1987, p. 164) celles de l'État, du marché, de la science ou de la technique. Ainsi, même si pour Levinas le problème de la justice sociale et de la distribution de la richesse est important, c'est surtout dans la critique de l'aliénation de l'homme qui est ancrée dans la raison instrumentale à l'époque de la technique, que l'on rencontre une aprobation de fond du marxisme. D'où l'affirmation de Levinas: "Le marxisme était beaucoup plus qu'une doctrine, plus qu'une idéologie. Il représentait le dévouement à l'autre homme. Il voulait sauver tous les hommes, ne pas les abandonner. Bien que le stalinisme ait compris tout cela, dans le marxisme, il y a cet élan vers l'autre homme." (LESCOURRET, 1994, p. 349). Ou, encore de manière plus directe, "L’Autre, c'est l'ouvrier." (LEVINAS, 1977b, p. 11, 53). Guy Petitdemange l'exprime à sa manière: "il y a un clin d'œil, les allusions presque toujours positives de Levinas à Marx et son mépris pour les Kautsky, Bernstein... Levinas y revient toujours sur deux points: l'importance de l'économique, de la vie économique, la nécessité d'aller toujours vers autrui 'les mains pleines'.” (PETITDEMANGE, 1998, p. 36).

Or, la coïncidence de pensée s'étend aussi à la Théologie de la libération ${ }^{7}$ et au mouvement de Mai 68, lui aussi d'inspiration marxiste. Levinas dira à

7 En ce qui concerne ce mouvement latino-américain, pour son principal représentant, Enrique Dussel, il s'agit de chercher à concrétiser l'abstraction de la chair souffrante des pauvres. Dussel part de la réalité socio-historique du pauvre comme travailleur exploité, comme un corps dépouillé. Cette altérité effectivement aliénée est celle de la misère concrète en Amérique latine. Dusssel, conformément à son origine chrétienne et hégélo-marxiste, s'emploie à donner une incarnation et une effectuation historique et pratique à cette négativité qu'est l'autre comme le pauvre vidé de tout. Dussel tente donc d'ouvrir la possibilité d'une politique de la libération qui dépasse l'horizon de la totalité, instituée au nom d'une ouverture dans l'ordre institué, toujours ouverte et toujours à venir et à faire advenir. Refusant ainsi l'enfermement de la totalité dominante dans l'abstraction éthérée de l'infini eschatologique, la praxis de la libération s'ouvre vers des futurs à jamais inachevés, construits pas les pratiques des oprimés suscitées par un mouvement de dépassement issu des souffrances, travaux et 
propos de la Théologie de la libération qu'il y a là "une intéressante tentative de revenir à l'esprit populaire sud-américain, une grande influence d'Heidegger d'autre part dans la manière, le rythme du dévelopement, dans la radicalité de questionnement. Je suis très heureux, très fier même, quand je trouve écho dans ce groupe. C'est une aprobation de fond. Cela veut dire que des gens ont vu 'ça' aussi." (LEVINAS, 1991b, p. 130). Par raport au mouvement de Mai 68 il écrira:

Dans la fulgurance de quelques instants privilégiés de 1968 -vite éteints par un langage aussi conformiste et aussi bavard que celui qu'il allait remplacerla jeunesse a consisté à contester un monde depuis longtemps dénoncé. Mais la dénonciation était devenue, depuis longtemps, littérature et clause de style. Certaines voix ou certains cris lui rendirent sa signification propre et irrécusable. La vague notion de l'authenticité -dont on abuse- prit ici un sens précis. La jeunesse est authenticité. Mais jeunesse définie par la sincérité qui n'est pas la brutalité de l'aveu et la violence de l'acte, mais aproche d'autrui, prise en charge du prochain, qui vient de la vulnérabilité humaine. (LEVINAS, 2012, p. 110).

Il y a aussi une aprobation de la pensée marxiste d'Ernest Bloch et surtout de la portée utopique de sa pensée. Bloch est un philosophe auquel Levinas dédie le texte intitulé Sur la mort dans la pensée d'Ernst Bloch publié en 1976 dans le recueil Utopie et marxisme selon Ernst Bloch.

Pourtant, l'aprobation plus profonde du socialisme libertaire doit surtout être cherchée dans le judaïsme libertaire de l'Europe centrale. Levinas était averti et fier du rôle fondateur et réformateur qu'avaient joué les juifs d'Europe centrale. Comme l'exprime bien Raphael Lellouche: "Levinas posé dans cette constellation philosophique n'est pas étranger au libertarisme juif d'Europe centrale." (LELLOUCHE, 2006, p. 105). Par ailleurs Levinas luimême déclare que:

Les engagements révolutionnaires d'une jeunesse formée dans les foyers de vie juive de l'Europe orientale qu'elle reniait pour le socialisme, et la dissidence précoce dans la résistance à la perversion stalinienne et à ses séquelles, et l'élan sioniste qui n’a jamais été séparable -et jusqu’à dans

luttes des exclus. L'horizon est ainsi celui d'une communauté historique possible, dans un processus infini de libération se construisant par des pratiques réformistes et révolutionnaires. La Théologie de la libération aura un impact sensible sur les mouvements d'émancipation du continent américain. À l'évidence, l'influence de Levinas chez Dussel était fort importante et parcourt la pensée du penseur naturalisé mexicain. Cf. DUSSEL, Enrique. Éthique communautaire. Traduction F. Guibal. Paris: CERF, 1990. 
l'État juif assiégé dès sa résurrection- du rêve messianique universel, ni de la subversion du discours prophétique défiant rois et seigneurs, éveillant les hommes engourdis par l'histoire à un ordre sans victoire ni hégémonies, sans guerres ni cruauté. (LESCOURRET, 1994, p. 349).

C'est à travers Rosenzweig que Levinas entretient un raport avec ce judaïsme, la préface de Totalité et infini témoigne de cette influence. C'est spécialement en raport avec Buber que la coöncidence avec le libertarisme juif peut être attestée. Levinas manifeste à plusieurs reprises l'influence de Buber à l'égard de sa pensée dans différents textes: spécifiquement dans Noms propres, les préfaces aux éditions allemandes de Totalité et infini ou dans Hors sujet; sans nommer l'importante correspondance entre les deux penseurs. Or, c'est surtout dans la préface de la traduction au français d'Utopie et socialisme que cette coïncidence doit être remarquée. Dans de longs passages, Levinas fait non seulement une présentation de la pensée politique de Buber, mais en filigrane, il semble faire aussi une présentation de sa propre pensée politique. Cela semble transparaître lorsqu'il déclare par exemple:

Le social, par contre, signifierait la "vie commune des hommes", leur compagnonnage, la présence de l'homme pour l'homme, sa proximité. Le socialisme consisterait à régénérer dans ce sens les "cellules" du tissu social altérées par la politique. (LEVINAS, 1977b, p. 10).

D'où,

La recherche de foyers sociaux multiples pour que la présence des personnes aux personnes soit une "présence réelle", d'où la décentralisation de l'ensemble pour éviter que l'organisation et l'administration ne viennent "étatiser", sous des règles abstraites et au nom de puissances anonymes, cet être-ensemble des hommes. (LEVINAS, 1977b, p. 10).

Or, ce qui est remarquable c'est que Levinas met en avant chez Buber une oposition à la philosophie politique hégélienne: "Comme s'il s'agissait de mettre en question la subordination de la société civile à l'État où, pour Hegel, l'humanité atteindrait l'universalité de la pensée et du vouloir crest-à-dire à la liberté. C'est à partir de l'idée de domination, de la coercition -ou, comme on dirait aujourd'hui de la répression- que Buber pense le raport politique entre hommes." (LEVINAS, 1977b, p. 9). D'où le fait que Levinas, pourtant dans un langage hégélien signale: 
En présence de certains actes de résistance et du martyr osés dans notre monde au nom de l'humain pur -de l'humain utopique- contre l'efficacité des pouvoirs et des puissances cette éthique affirme son statut objectif, se montre Wirklichkeit, réalité efficace, ne se laisse plus refouler parmi les impuissances de "belles âmes" ou de "conscience malheureuse". (LEVINAS, 1977b, p. 10-11).

Mais qu'y a-t-il d'hégelien, paradoxalement, dans cette oposition éthique de la société civile à l'État ? Et n'est-ce pas cette entrée nécessaire de l'État chez Levinas qui permet de mesurer toute la distance de Levinas à l'égard de Buber, de Rosenzweig et de la pensée libertaire ? Alors, comment comprendre cette contradiction?

Or, c'est dans cette même préface que Levinas s'autorise par la lecture de Buber à se donner une voie de sortie:

Mais on peut se demander si le dépérissement de l'État ne s'ajourne pas ainsi indéfiniment. Un exemple, seul et unique, d'une société socialiste dont la réalisation n'aura pas échoué: les fermes collectives -les kibboutzimsur le sol de la terre d'Israël [...]. Mais les kibboutzim eux-mêmes sont qualifiés par Buber de non échecs, plutôt que de succès. Il y aurait donc entre la société et l'État une redoutable dialectique. Plutôt que simple effet des abus de pouvoirs, les crises n'apartiennent-elles pas à l'essence même du corps collectif ? Une question et une note pessimiste dans toute cette évocation de l'utopie socialiste et du socialisme tout court. (LEVINAS, 1977b, p. 10).

Pourtant poursuit Levinas (1977b, p. 10):

À moins que cette évocation, la recherche toujours renaissante d'une société où doit s'accomplir l'être ensemble des hommes, la résistance oposée à l'oubli de ce devoir-être utopique au sein même des structures de l'État s'érigeant en fin en soi, la résurgence de la conscience contre l'État altérant les relations sociales - ne soient elles-mêmes des événements objectifs. Événements marquant dans la dialectique société-État, le moment de la morale limitant la politique, moment indétournable et inoubliable.

C'est ce passage qui marque toute la proximité ainsi que toute la distance à l'égard de Buber, celui qui peut peut-être nous donner la clé pour finir de saisir la pensée politique de Levinas. 
BALBONTIN-GALLO, C. Levinas and Liberarian Socialism. Trans/form/ação, Marília, v. 44, n. 4, p. 79-100, Out./Dez., 2021.

\begin{abstract}
In Autrement qu'être Levinas introduces the notion of "an-archy", not only to refer to an immemorial order of meaning in the trace of the other in oneself, but also in a political sense that claims a disorder or even a power of revolt stemming from the ethical sociality that the experience of the face awakens. Henceforth, the establishment of the order of political meaning no longer makes it possible to embrace or exhaust the sociality resulting from this intersubjective experience. In this anarchic demand for the sociality of the human Levinas thus joins some presupositions of socialism and libertarian Judaism in Europe. It is therefore a question of identifying possible points of raprochement and crossroads, which will apear in a way that is often unexpected for certain Levinassian orthodoxy.
\end{abstract}

Keywords: Socialism. Anarchy. Judaism. Libertarian. Levinas.

\title{
RÉFÉRENCES
}

BOUSQUET, Joë. Anthologie. Paris: Rivages, 1981.

BUBER, Martin. Zion, der Statt und die Menschheit. Bemerkungen zu Hermanns Cohen Antwort. Berlin: Der Jude, I, 1916.

CARLYLE, Thomas. Signs of times. Edinburgh Review, Edinburgh, n. 49, 1829.

CHALIER, Catherine. Exposition dans le colloque français d'intellectuels juifs. In: ALPÉRIN, Jean; HANSSON, Nelly. Difficile justice. Dans la trace d'Emmanuel Levinas. Paris: Albin Michel, 1998.

DROZ, Jacques. Le romantisme politique en Europe. Paris: Armand Collin, 1963.

ENGELS, Friedrich. The condition of the working class in England in 1884. New York: J. W. Lovell, 1987.

HEGEL, Georg W. F. L'Encyclopédie des sciences philosophiques. Troisième partie: Philosophie de l'esprit. Traduction Bourgeois. Paris: Vrin, 1988.

HEGEL, Georg W. F. Principes de la philosophie du droit. Traduction J. F. Kervegan. Paris: PUF, 1998.

HENNING, Günther. Walter Benjamin zwischen Marxismus und Theologie. Freiburg: Olter Walter, 1974.

LELLOUCHE, Raphael. Difficile Levinas. Peut-on ne pas être levinassien? Paris: Eclat, 2006.

LESCOURRET, Marie-Anne. Emmanuel Levinas. Paris: Flammarion, 1994. 
LEVINAS, Emmanuel. Totalité et infini. La Haye: Martinus Nijhoff, 1961.

LEVINAS, Emmanuel. Autrement qu'être ou au-delà de l'essence. La Haye: Martinus Nijhoff, 1974.

LEVINAS, Emmanuel. Noms propres. Paris: Fata Morgana, 1976.

LEVINAS, Emmanuel. Préface de Socialisme et utopie. In: BUBER, Martin. Socialisme et utopie. Paris: Aubier, 1977a.

LEVINAS, Emmanuel. Judaïsme et révolution. In: LEVINAS, Emmanuel. Du sacre au saint. Paris: Minuit, $1977 \mathrm{~b}$.

LEVINAS, Emmanuel. L'État de César et l'État de David. In: LEVINAS, Emmanuel. L’au-delà du verset. Paris: Minuit, 1982.

LEVINAS, Emmanuel. Temps et l'autre. Paris: PUF-Quadrige, 1983.

LEVINAS, Emmanuel. Paix et proximité. In: ROLLAND, Jacques (ed.). Emmanuel Levinas. Les Cahiers de La nuit surveillée. Lagrasse: Verdier, 1984.

LEVINAS, Emmanuel. Les droits de l'homme et les droits d'autrui. In: LEVINAS, Emmanuel. Hors sujet. Paris: Fata Morgana (poche), 1987.

LEVINAS, Emmanuel. Les droits de l'homme sont-ils un nouvel impérialisme ? Le Nouvel Observateur, Paris, n. 2, oct. 1990 (Collection Dossiers).

LEVINAS, Emmanuel. Transcendance et hauteur. In: CHALIER, Catherine; ABENSOUR, Miguel (ed.). Cahier de l'Herne Emmanuel Levinas. Paris: L'Herne, 1991a. p. 97-119.

LEVINAS, Emmanuel. Entre nous. Essai sur penser à l'autre. Paris: Grasset, $1991 \mathrm{~b}$.

LEVINAS, Emmanuel. De Dieu qui vient à l'idée. Paris: Vrin, 1992a.

LEVINAS, Emmanuel. Entretien avec Roger Pol-Droit journal "Le monde $N^{o}$ 14.725”, 2 juin $1992 b$.

LEVINAS, Emmanuel. Dieu, la mort et le temps. Paris: Grasset, 1993.

LEVINAS, Emmanuel. En découvrant l'existence avec Husserl et Heidegger. Paris: Vrin, 2001.

LEVINAS, Emmanuel. Difficile liberté. Paris: Albin Michel, 2006.

LEVINAS, Emmanuel. Humanisme de l'autre homme. Paris: Fata Morgana (poche), 2012.

LUKACS, György. Histoire et conscience de classes. Paris: Minuit, 1960.

MARX, Karl. Manifeste du parti communiste. Paris: BNF-Bibebook, 1895.

PETITDEMANGE, Guy. La notion paradoxale d'histoire. In: FROGNEUX, Nathalie; MIES, Françoise (ed.). Emmanuel Levinas et l'histoire. Paris: CERF, 1998. 
POIRIER, François. Emmanuel Levinas. Qui êtes-vous ? Paris: Actes Sud, 2006. ROLLAND, Jacques. Parcours de l'autrement. Paris: PUF, 2000.

ROSENZWEIG, Franz. Hic et ubique! In: ROSENZWEIG, Franz. Kleinere Schriften. Berlin: Schoken, 1937.

ROSENZWEIG, Franz. Étoile de la Rédemption. Paris: Seuil, 1982.

ROUSSEAU, Jean Jacques. Discours sur l'origine et les fondements de l'inégalité parmi les hommes. Paris: Bordas, 1987.

WEBER, Max. Le savant et le politique. Paris: UGE, 1963. 